Physics in Collision (PIC 2013)

International Journal of Modern Physics: Conference Series

Vol. 31 (2014) 1460298 (5 pages)

(C) The Authors

DOI: $10.1142 / \mathrm{S} 2010194514602981$

\title{
MRPC detector for the upgrade of BESIII E-TOF
}

\author{
Rong-Xing Yang, Yong-Jie Sun and Cheng Li* \\ Department of Modern Physics, University of Science \\ and Technology of China, Hefei 230026, Anhui, China \\ *licheng@ustc.edu.cn
}

Published 15 May 2014

\begin{abstract}
An end-cap Time-of-Flight (E-TOF) system with higher granularity and intrinsic time resolution of better than $50 \mathrm{ps}$ will extend the $\mathrm{K} /$ pion separation (2 sigma) pT range to $1.4 \mathrm{GeV} / \mathrm{c}$ and enhance the physics capability of Beijing Spectrometer (BESIII). A R\&D work was carried out aiming at upgrading the current BESIII E-TOF with the Multi-gap resistive Plate Chamber (MRPC) detector. The latest best test for the prototype MRPC, together with the custom designed Front End Electronics (FEE) and TDC boards, was performed at the BEPC E3 line in July 2012. The test results show that time resolution of less than $50 \mathrm{ps}$ can be achieved with such a system.
\end{abstract}

Keywords: Multi-gap resistive plate chamber; BESIII; End-cap TOF.

\section{Introduction}

The MRPC detector is a good candidate for large area TOF system with very good time resolution, high efficiency and relatively low cost per channel..$^{1,2}$ It has been widely used as TOF detector in many experiments, such as LHC/ALICE, ${ }^{3}$ RHIC/STAR. ${ }^{4}$ The BESIII ${ }^{5}$ is a high precision general-purpose detector designed for the high luminosity in the $\tau$-charm energy region at the upgraded Beijing Electron-Positron Collider (BEPCII). ${ }^{6}$ A MRPC-based TOF with 50 ps intrinsic time resolution is estimated to extend the BESIII $\mathrm{K} / \pi$ separation $(2 \sigma)$ momentum range to $1.4 \mathrm{GeV} / \mathrm{c}$ at BESIII end-cap. A proposal was raised to upgrade the current BESIII ETOF with the MRPC technology and readout electronics, aiming at the 50 ps time resolution.

\section{Design}

In the conceptual design, each BESIII end-cap ring will be fully covered by 36 trapezium MRPC modules, as shown in Fig. 1. The MRPC module to be used on

This is an Open Access article published by World Scientific Publishing Company. It is distributed under the terms of the Creative Commons Attribution 3.0 (CC-BY) License. Further distribution of this work is permitted, provided the original work is properly cited.

${ }^{*}$ Corresponding author. 


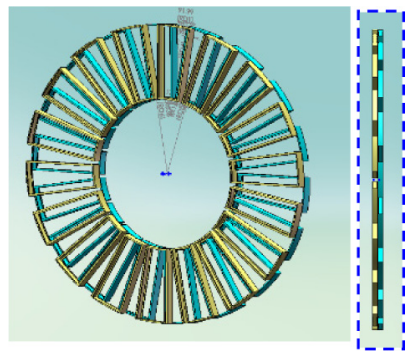

Fig. 1. The schematic of BESIII MRPC/ETOF.
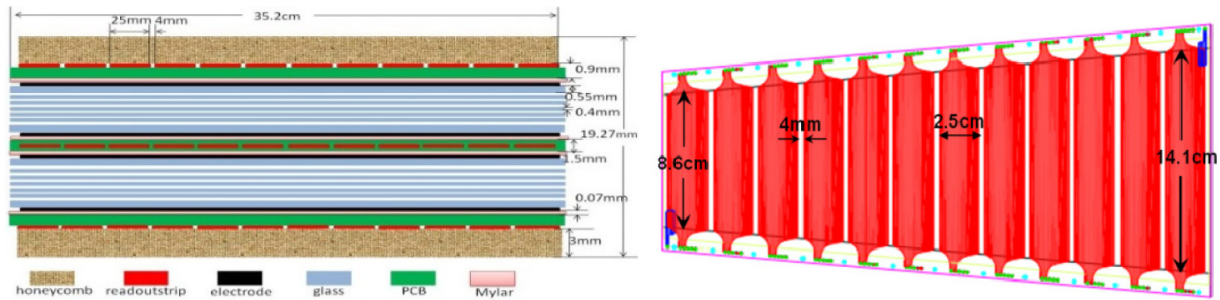

Fig. 2. Left: The side-view of the MRPC structure. Right: the readout pattern.

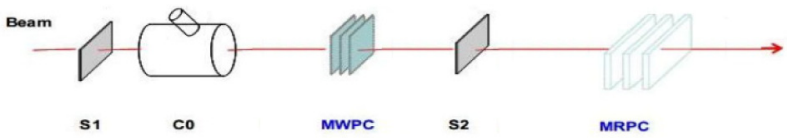

Fig. 3. The setup at BEPC E3 line.

BESIII E-TOF has 12 gas gaps of $0.22 \mathrm{~mm}$ thick, arranged in two stacks. Thin floating glass with bulk resistivity $\sim 10^{13} \Omega / \square$ is used as the resistive plate. The signals are picked up by 12 strips with different length and read out from both ends of each strip. The structure of the MRPC is plotted in Fig. 2.

\section{Test}

The test was performed at BEPC E3 line. The setup of the test is shown in Fig. 3. There MRPC detectors are arranged along the beam line close to each other. The FEE board is custom designed with NINO ASIC chips. The time jitter of the FEE is tested $\sim 10$ ps. The custom designed far-end electronics is based on HPTDC chips with Leading- and Trailing-edge recording capability. Thus, the TOT (TimeOver-Threshold) of the signal can be used for the slewing correction instead of the traditional T-A correction. The secondary beam contains pions and protons. They can be distinguished clearly by the flight time between scintillators S1 and S2, as shown in Fig. 4. 


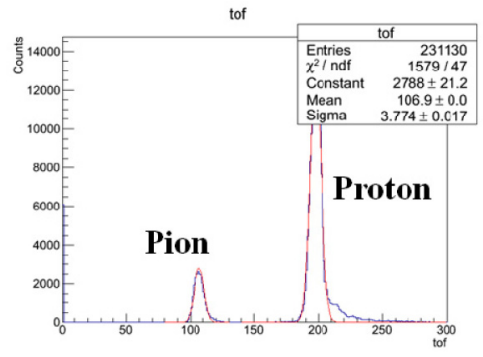

Fig. 4. The PID with TOF between S1 and S2.
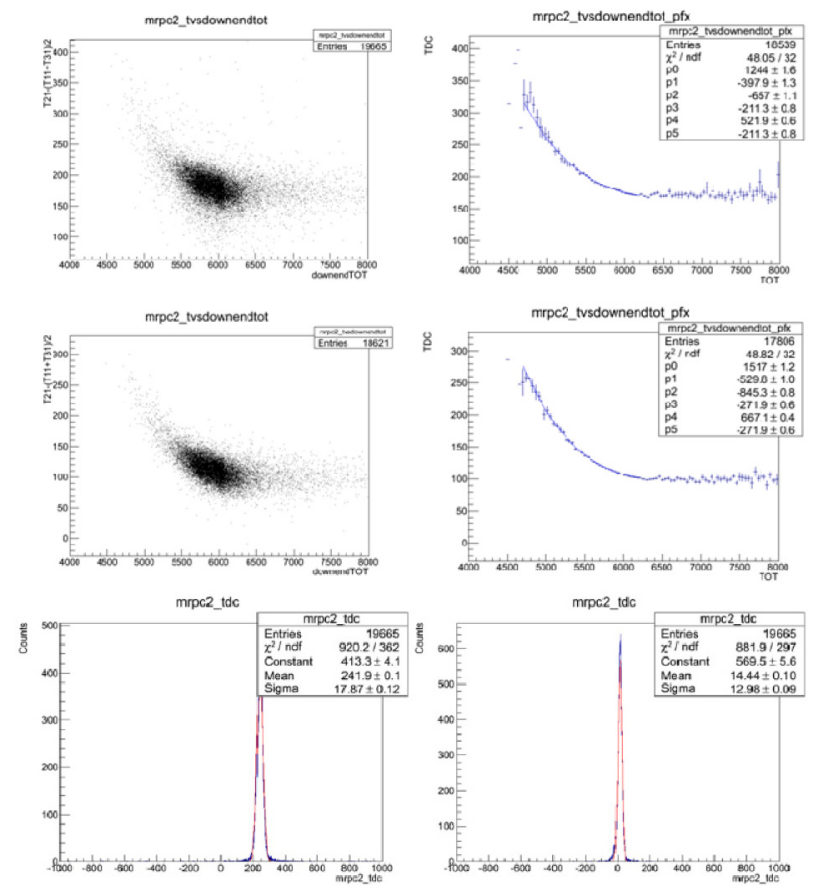

Fig. 5. The slewing correction and the time spectrum.

The MRPC was first tested with pion samples around $600 \mathrm{MeV}$ which can be considered as MIPs. The slewing correction was made with T-TOT correlation. For each MRPC, the other two modules can be used as reference time (T0). Correcting the time circularly until stable correction relation achieved, the time resolution of each MRPC can be solved. Figure 5 shows the slewing correction and the time spectrum before and after the correction.

The performance of the MRPC is also analyzed with proton samples which is the main contents of the beam. Since proton at such momentum is not MIP, the time resolution is much better, as summarized in Table 1. 
Table 1. The time resolution achieved with pion and proton samples at different momentum.

\begin{tabular}{lcccc}
\hline \multicolumn{2}{c}{ Momentum } & $500 \mathrm{MeV}$ & $600 \mathrm{MeV}$ & $800 \mathrm{MeV}$ \\
\hline Pion sample & MRPC \#1 & $56 \mathrm{PS}$ & $47 \mathrm{PS}$ & $45 \mathrm{PS}$ \\
& MRPC \#2 & $51 \mathrm{PS}$ & $48 \mathrm{PS}$ & $40 \mathrm{PS}$ \\
& MRPC \#3 & $46 \mathrm{PS}$ & $48 \mathrm{PS}$ & $45 \mathrm{PS}$ \\
Proton sample & MRPC \#1 & $29 \mathrm{PS}$ & $32 \mathrm{PS}$ & $36 \mathrm{PS}$ \\
& MRPC \#2 & $28 \mathrm{PS}$ & $30 \mathrm{PS}$ & $35 \mathrm{PS}$ \\
& MRPC \#3 & $31 \mathrm{PS}$ & $31 \mathrm{PS}$ & $35 \mathrm{PS}$ \\
\hline
\end{tabular}

Table 2. The time resolution on different incident positions along a selected strip.

\begin{tabular}{lllllll}
\hline MRPC \#2 @600MeV & \multicolumn{5}{c}{ Strip center $\rightarrow$ Strip end } \\
\hline Pion & & 48 PS & & 49 PS & 49 PS & 47 PS \\
Proton & 29 PS & 27 PS & 26 PS & 29 PS & 31 PS & 36 PS \\
\hline
\end{tabular}

Table 3. The time resolution under different applied High Voltage.

\begin{tabular}{lccc}
\hline$@ 600 \mathrm{MeV}$ & $\pm 7250 \mathrm{~V}$ & $\pm 7300 \mathrm{~V}$ & $\pm 7500 \mathrm{~V}$ \\
\hline Pion & $48 \mathrm{PS}$ & $48 \mathrm{PS}$ & $46 \mathrm{PS}$ \\
Proton & $30 \mathrm{PS}$ & $29 \mathrm{PS}$ & $30 \mathrm{PS}$ \\
\hline
\end{tabular}

The performance uniformity along the strip is checked by moving one of the MRPC modules along the strip direction. As shown in Table 2, no position dependence is found. Table 3 shows the time resolution under different applied High Voltage, which indicates the stable performance profited from the long plateau of MRPC.

\section{Summary}

We successfully design, built and test the MRPC prototype with 12 gas gap structure and double-end readout strips for BESIII ETOF. The proposed performance has been achieved. The time resolution including the custom designed electronics is $\sim 50$ ps for MIPs. The detector shows stable performance at different position and working HV. The systematic construction project has been approved and will start soon.

\section{References}

1. M. C. S. Williams, E. Cerron-Zeballos et al., Nucl. Instr. and Meth. A434 (1999) 362372 .

2. Akindinov, A. Alici et al., Nucl. Instr. and Meth. A602 (2009) 709-712. 
3. ALICE Collaboration, Addendum to ALICE TDR 8, CERN/LHCC/2002-16, April 2002.

4. STAR TOF Collaboration, Proposal for a large area time of flight system for STAR (2003).

5. M. Ablikim et al., Nucl. Instr. and Meth. A614 (2010) 345-399.

6. D. Asner et al., Ins. J. Mod. Phys. A24S1 (2009) 499-502. 